\title{
The Scientific Impact of the
}

\section{Vera C. Rubin Observatory's Legacy Survey of Space and Time (LSST) for Solar System Science}

\author{
Vera C. Rubin Observatory LSST Solar System Science Collaboration, ${ }^{1}$ \\ R. Lynne Jones, $, 2,3, *$ Michelle T. Bannister, ${ }^{4}$ Bryce T. Bolin, ${ }^{5}$ \\ Colin Orion Chandler, ${ }^{6}$ Steven R. Chesley, ${ }^{7}$ Siegfried Eggl, ${ }^{2,}, 3$ \\ Sarah Greenstreet, ${ }^{2}$ Timothy R. Holt $,{ }^{8}, 9$ Henry H. Hsieh, ${ }^{10,}, 11$ Želuko Ivezić, ${ }^{2,}, 3$ \\ Mario Jurić, ${ }^{2,3}$ Michael S. P. Kelley, ${ }^{12}$ Matthew M. Knight, ${ }^{13}, 12$ \\ Renu Malhotra, ${ }^{14}$ William J. Oldroyd,${ }^{6}$ Gal Sarid, ${ }^{15}$ Megan E. Schwamb,${ }^{16}$ \\ Colin Snodgrass, ${ }^{17}$ Michael Solontoi, ${ }^{18}$ and David E. Trilling ${ }^{6,19}$ \\ ${ }^{1}$ Vera C. Rubin LSST Science Collaborations \\ ${ }^{2}$ DIRAC Institute and Department of Astronomy, University of Washington, Seattle, Washington, USA \\ ${ }^{3}$ Vera C. Rubin Observatory, Tucson, Arizona, USA \\ ${ }^{4}$ School of Physical and Chemical Sciences - Te Kura Matū, University of Canterbury, New Zealand \\ ${ }^{5}$ IPAC, California Institute of Technology, Pasadena, CA, USA \\ ${ }^{6}$ Department of Astronomy $\& 3$ Planetary Science, Northern Arizona University, Flagstaff, AZ, USA \\ ${ }^{7}$ Jet Propulsion Laboratory, California Institute of Technology, Pasadena, CA, USA \\ ${ }^{8}$ Centre for Astrophysics, University of Southern Queensland, Queensland, Australia \\ ${ }^{9}$ Department of Space Studies, Southwest Research Institute, Boulder, Colorado, USA \\ ${ }^{10}$ Planetary Science Institute, Tucson, Arizona, USA \\ ${ }^{11}$ Academia Sinica Institute of Astronomy and Astrophysics, Taipei, Taiwan \\ ${ }^{12}$ University of Maryland, College Park, Maryland, USA \\ ${ }^{13}$ United States Naval Academy, Annapolis, Maryland, USA \\ ${ }^{14}$ Lunar and Planetary Laboratory, The University of Arizona, Tucson, USA \\ ${ }^{15}$ SETI Institute, Mountain View, CA, US \\ ${ }^{16}$ Queen's University Belfast, Belfast, UK \\ ${ }^{17}$ Institute for Astronomy, University of Edinburgh, Royal Observatory, Edinburgh, UK \\ ${ }^{18}$ Monmouth College, Monmouth, Illinois, USA \\ ${ }^{19}$ Lowell Observatory, Flagstaff, AZ, USA
}

(Dated: July 15, 2020)

* Corresponding author email: lynnej@uw.edu 


\section{INTRODUCTION}

Vera C. Rubin Observatory will be a key facility for small body science in planetary astronomy over the next decade. It will carry out the Legacy Survey of Space and Time (LSST), observing the sky repeatedly in $u, g, r, i, z$, and $y$ over the course of ten years using a $6.5 \mathrm{~m}$ effective diameter telescope with a 9.6 square degree field of view, reaching approximately $r=24.5 \mathrm{mag}(5-\sigma$ depth$)$ per visit. The resulting dataset will provide extraordinary opportunities for both discovery and characterization of large numbers (10-100 times more than currently known) of small solar system bodies, furthering studies of planetary formation and evolution. This white paper summarizes some of the expected science from the ten years of LSST, and emphasizes that the planetary astronomy community should remain invested in the path of Rubin Observatory once the LSST is complete.

\section{THE LSST AS A SMALL-BODY DISCOVERY MACHINE}

Discovery and census of objects in the solar system is one of the four foundational science cases for the LSST (Ivezić et al. 2019). The suitability of the final LSST cadence for the discovery and characterization of solar system objects will therefore be a high-priority consideration in its selection. A solar system object observed by the LSST will be identified as such with $95 \%$ efficiency (on average) if it was detected on at least three nights within a window of 15 days, with a minimum of two visits per night (Ivezić et al. 2019). Realistic simulations performed using the Moving Object Processing System (MOPS; which was developed by the Pan-STARRS survey and adopted by LSST) show $>99 \%$ linking efficiency across all classes of solar system objects (Denneau et al. 2013), and at least 93\% efficiency for Near-Earth objects (NEOs; Vereš \& Chesley 2017a,b) using this criterion. Newly discovered objects will be reported to the Minor Planet Center, generally within 24 hours of discovery.

The LSST alert stream will identify all transient objects in each image, including any small body detections or trailed objects, and distribute alerts within 60 seconds of shutter closure. In total, the LSST will obtain approximately 1.8 billion observations of 5.5 million objects over 10 years. Data will be automatically reduced and delivered with systematiclimited astrometric and photometric precisions of 10 mas and 0.01 mag, respectively. Rubin Observatory will be by far the most prolific discovery and characterization machine of small solar system bodies throughout the 2020s.

Table 1 summarizes estimated LSST discovery yields for several major solar system populations. Most discoveries will occur during the first few years of the survey (Figure 1). A significant fraction of these objects will receive multi-band observations suitable for measuring colors and lightcurves. Beyond these major populations, the LSST will also discover an order of magnitude more interstellar objects, active asteroids, Centaurs, planetary Trojans, temporarily captured objects (i.e., "mini-moons"), Hildas, and inner Oort Cloud objects.

\section{SCIENCE ENABLED BY LSST}

\subsection{Near-Earth objects}

The LSST plays a crucial role in achieving the congressional target of discovering $90 \%$ of all potentially hazardous asteroids (PHAs) with $H \approx 22$ (Vereš \& Chesley 2017a; Jones et al. 2018). Current predictions suggest that the LSST alone could be responsible for a five-fold 
Table 1. Small-body population numbers as of today (7/2020; JPL Small-Body Database) and after LSST (approximate predicted LSST results based on simulations).

\begin{tabular}{lcc}
\hline \hline Population & Currently known (approximate) & LSST discoveries (predicted) \\
Near-Earth objects & 23000 & 100000 \\
Main Belt asteroids & 856000 & 5000000 \\
Jovian Trojans & 8000 & 280000 \\
Trans-Neptunian objects & 3500 & 40000 \\
Comets & 4000 & 10000 \\
Interstellar objects & 2 & $>10$ \\
\hline
\end{tabular}

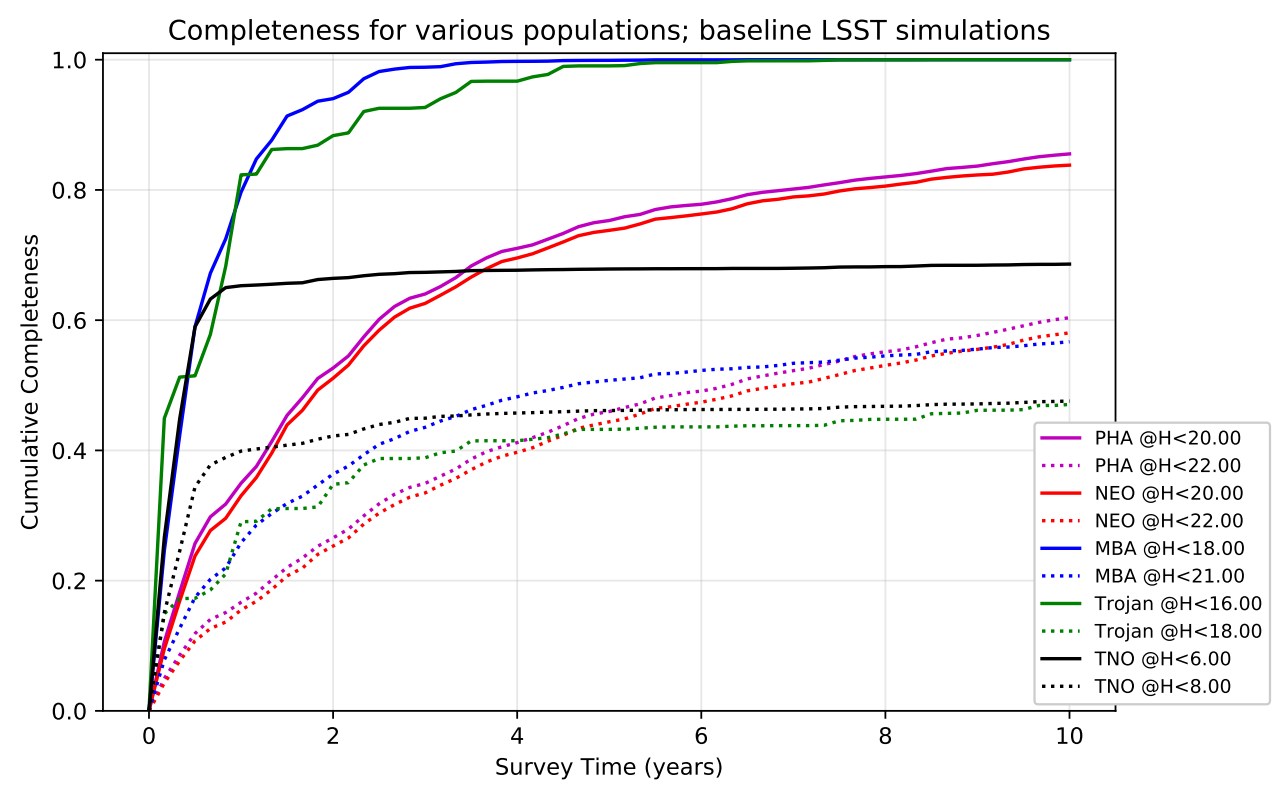

Figure 1. LSST discovery completeness as a function of time, for various modeled solar system populations (Granvik et al. 2018; Grav et al. 2011; Petit et al. 2011; Kavelaars et al. 2009). Cumulative completeness is reported for two different absolute magnitude $(H)$ values; one near the bright end of the population (solid lines) and one near the $50 \%$ completeness level (dashed lines). These follow similar tracks over time, showing that most objects are discovered early in the survey.

increase in the total number of known NEOs by the end of the survey (Table 1), detecting on the order of 100000 NEOs at a range of sizes down to $H<25$ mag.

The LSST's excellent astrometric precision $(\sigma \approx 10$ mas $)$ is expected to facilitate detections of non-gravitational forces acting on orbits, including the Yarkovsky effect, solar radiation pressure, outgassing, and collisions. Understanding how these effects influence NEO trajectories is critical to our ability to precisely predict future impacts (Farnocchia et al. 2013).

The timely processing and publication of NEO observations allows impact monitoring services to provide advance notice of close approaches and potential impacts. This facilitates critical characterization efforts including radar, spectroscopic, and light curve observations. Variations in the brightness of NEOs can also yield clues on NEO disruption mechanisms at small perihelion distances (Granvik et al. 2016), which can then be utilized to probe NEO internal structure and test dynamical models, both critical for effective planetary defense. 
Yearly releases of NEO catalogs exclusively based on LSST observations enable unbiased population predictions of unprecedented quality. Unbiased populations can be used to quantify the long-term impact flux of NEOs as a function of size (Granvik et al. 2018). The orbital, absolute magnitude, and taxonomy distributions within the NEO population derived from the LSST data can help identify correlations between taxonomy and orbital properties for NEOs, as well as determine the orbital distribution of objects down to ten meters in size.

\subsection{Main Belt asteroids}

Main Belt asteroids (MBAs) represent important compositional and dynamical tracers of the solar system's formation and evolution. Their orbital element distribution, size-frequency distributions, and total mass provide key constraints for planetary migration models like the Nice Model and the Grand Tack Model (Morbidelli et al. 2010; Walsh et al. 2011; Yoshida et al. 2019), while taxonomic classifications provide additional constraints on those models and also enable the tracing of more recent dynamical and physical evolution (e.g., DeMeo \& Carry 2014). The LSST will discover an order of magnitude more MBAs than are currently known, providing new insights into solar system formation and evolution.

Beyond discovery, the multi-band, multi-epoch observations from the LSST $(\sim 200-300$ photometric measurements per object for over 5 million MBAs during its 10-year survey; Section 5 in LSST Science Collaboration et al. 2009) will enable detailed physical studies. Precise multi-band optical colors will enable robust and efficient taxonomic classification of MBAs (Carvano et al. 2010), and detailed studies of asteroid families (e.g., Ivezić et al. 2002; Parker et al. 2008), where color precisions on the order of 0.01 mag for sufficiently bright asteroids are expected to be achievable by the LSST. When combined with infrared data, optical albedos can be estimated and used to improve taxonomic and family classifications (Mainzer et al. 2012), and to derive physical size estimates (Moeyens et al. 2020; Ivezić \& Ivezić 2020).

\subsection{Short-period comets and active asteroids}

Active objects are small solar system bodies that exhibit any type of mass loss, whether driven by ice sublimation, impact disruption, rotational destabilization, or other mechanisms. These bodies inform us about the distribution of volatiles throughout the solar system and hold clues about solar system formation. They include Jupiter-family comets (JFCs), Oort Cloud comets (Section 3.5), active asteroids (Jewitt et al. 2015; Snodgrass et al. 2017), active Centaurs (Jewitt 2009), and active interstellar objects (Section 3.6).

An order of magnitude increase in the known JFC population from the LSST will facilitate improved modeling of their source regions and evolutionary processes (Brasser \& Wang 2015) and enable large-scale photometric and spectroscopic follow-up studies that will improve our ability to identify and characterize distinct taxonomic classes (Cochran et al. 2015). Meanwhile, only about 30 active asteroids are currently known, making them difficult to characterize on a population-scale basis, given the diversity exhibited by the population thus far (Jewitt et al. 2015). The identification of many more active asteroids by the LSST will both enable population-level studies, such as efforts to ascertain the rates of rotational and impact disruptions in the asteroid belt (Denneau et al. 2015; McLoughlin et al. 2015), and increase the number of individual objects for targeted studies. 
The LSST's 10-year timescale is well-suited for the study of active asteroids and shortperiod comets, as all active asteroids and about half of all known JFCs have orbital periods of less than 10 years. LSST should produce data sets for a large number of these objects around at least one full orbit, providing at least some of the following: constraints on the initiation/cessation and seasonal variation of activity (A'Hearn et al. 1995), coarse sampling of outbursts as functions of orbit position (Ishiguro et al. 2016), nucleus size estimates (Fernández et al. 2013), and rotational lightcurves to constrain rotation periods, axis ratios, and pole orientations, and identify binarity or higher-order multiplicity (Lamy et al. 2004; Kokotanekova et al. 2017). The LSST's well-sampled data set will also enable systematic studies of active object nuclei before and after active events, helping us to better understand small body interiors, space weathering, and the effects of cometary outgassing (e.g., Bodewits et al. 2014). Extending the initial 10-year survey would permit many comets to be observed over multiple orbits, investigating the secular evolution of cometary activity on an unprecedented scale (e.g., Kelley et al. 2019; Nesvorný et al. 2017).

\subsection{Trans-Neptunian Objects}

Trans-Neptunian Objects (TNOs), Centaurs, and Scattered Disk Objects (SDOs) provide key insights into planetary formation and evolution, recording the imprint of the formation and migration of the giant planets in their orbital distributions and physical properties. Decoding these imprints has been hindered by the lack of sufficiently large population samples with high precision orbits and well understood discovery circumstances. The LSST will discover on the order of tens of thousands of TNOs, SDOs and Centaurs, and will deliver these objects with extremely precise orbits (due to high-precision astrometry spanning multiple years). Many of the objects will have multi-color photometry suitable for calculating colors and lightcurves for rough composition and rotation rate distributions.

The discovery, orbital classification, and physical characterization of large numbers of TNOs will help address the origin of the cold classical Kuiper belt, determining if these objects are primordial or were implanted into these orbits early in the history of the solar system, and ascertain its connections with other populations, such as Oort Cloud comets, Centaurs, and planetary Trojans (e.g., Nesvorný 2018).

Due to its depth and wide sky coverage, the LSST will discover many more high perihelia, large semi-major axis TNOs, such as inner Oort cloud objects (i.e. Sedna-like objects) and "extreme" TNOs (with $q>40$ AU and $a>150-250 \mathrm{AU}$ ), than the $<20$ that are currently known. A larger sample with well understood discovery circumstances will help ascertain whether their source is related to a hypothesized distant planet (Trujillo \& Sheppard 2014; Batygin et al. 2019), Neptune migration, stellar perturbations, or some other mechanism (e.g., Kavelaars et al. 2020), and also whether their observed orbital alignment is real (possibly indicating the presence of a distant planet) or the result of observational bias (e.g., Trujillo 2020; Brown \& Batygin 2019; Shankman et al. 2017). Additionally, the LSST may directly detect a distant planet if it is sufficiently bright and within the survey footprint, otherwise ruling out its presence in $61 \%$ of the sky (Trilling et al. 2018). A potential survey extension using deeper (10 minute, $r \approx 26$ ) exposures would increase the survey sensitivity for more distant and smaller TNOs. 


\subsection{Oort cloud comets}

As the most distant solar system population, the Oort Cloud and its structure reflect the early evolution of the solar system and that of the local Galactic environment (Heisler \& Tremaine 1986; Kaib et al. 2011). Oort Cloud objects are beyond current observational limits in-situ, however, and thus Oort Cloud comets (OCCs) provide our only opportunities to probe the physical and dynamical properties of these objects. The LSST is expected to discover thousands of OCCs (Solontoi et al. 2010), with generous photometric orbital coverage thanks to the survey's exceptional single-visit sensitivity limit.

The discovery of distant OCCs, with perihelion distances, $q,>10 \mathrm{au}$, enables follow-up characterization of a population that has likely never been closer to the Sun than Jupiter, and therefore in an earlier evolutionary phase than OCCs with $q<5$ au. At this stage, comet orbits are less altered by planetary perturbations and non-gravitational forces, and offer a rare opportunity to place constraints on the Oort cloud population. Furthermore, the discovery of comets with perihelia beyond 15 au directly tests the existence of the inner Oort cloud (Vokrouhlický et al. 2019). Currently, no comets have been discovered with $q>12$ au (as of July 2020; Giorgini et al. 1996), making this an important discovery space for LSST.

Early detection of inbound OCCs provides us with the opportunity to study the active behavior of this population. A long standing problem with our current knowledge of the OCC population is the apparent paucity of long-period objects, the so-called long-period comet fading problem. Ad-hoc models of OCC fading (e.g., splitting, disintegration, depletion of volatiles) have succeeded in addressing the missing comets (Wiegert \& Tremaine 1999), but physically-motivated explanations are still needed (Vokrouhlický et al. 2019). With an increased discovery rate and broad orbital coverage of OCCs, LSST will enable the study of key aspects of OCC evolution, such as a statistical description of when comets begin phases of activity, undergo fragmentation or disintegration, and the follow-up study of these events.

\subsection{Interstellar objects}

Detections of the first two known interstellar objects, 1I/'Oumuamua in 2017 (e.g., Meech et al. 2017) and 2I/Borisov (e.g., Fitzsimmons et al. 2019) in 2019, have given the first glimpses of macroscopic material from other solar systems and revealed that the range of properties of small bodies is broader than previously known. Based on the discoveries of 1I and 2I, it now seems reasonable to estimate that the LSST will discover on the order of ten interstellar objects (ISOs) during its first ten years. While each newly discovered ISO will undoubtedly be studied in great detail, collective statistics for the population must reach critical mass to enable meaningful tests of planet formation mechanisms and planetesimal ejection rates in other star systems. Continued surveying by Rubin Observatory beyond the initial 10 year LSST would be highly beneficial to the study of ISOs by improving population statistics. A future survey extension with deeper imaging would increase the survey volume to allow more robust investigation of the ISO size distribution and reveal whether it is reflective of these objects' formation or evolution, insight critically needed in order to understand the context for 1I and 2I (cf. 'Oumuamua ISSI Team et al. 2019).

\section{CONNECTIONS WITH MISSIONS AND OTHER FACILITIES}


Previous studies combining SDSS asteroid data with data from other ground and spacebased sources forecast strong synergistic value of the LSST dataset (e.g., Parker et al. 2008; Carvano et al. 2010; Mainzer et al. 2012). Both the multi-color nature and well-sampled light curves for an unprecedentedly large sample of small bodies will be exceedingly valuable for other missions. Follow-up of rare objects discovered by the LSST will be a high priority for facilities as the James Webb Space Telescope and 30m-class telescopes like the Thirty-Meter Telescope, Giant Magellan Telescope, and Extremely Large Telescope. Deep coordinated searches with missions such as the Roman Space Telescope would open new ways to probe small and cold solar system objects. Meanwhile, combining data from the LSST and the upcoming NEO Surveillance Mission spacecraft will allow determination of diameters and albedos for an unprecedented number of solar system objects (cf. Mainzer et al. 2019), while LSST lightcurves will be essential for assembling data collected over several days by NASA's SPHEREx mission, which is expected to collect $0.75-5.0 \mu \mathrm{m}$ spectral data for $\sim 200,000$ asteroids, into self-consistent spectra for each object.

Discoveries from the LSST will help to enhance the upcoming NASA Discovery missions Lucy (launch date 2021), targeting seven Jovian Trojans, and Psyche (launch date 2022), targeting potentially metallic asteroid (16) Psyche. These can offer opportunities to extend the Lucy mission with new targets of opportunity (Schwamb et al. 2018b), while observations of larger populations of similar asteroids will add context to the results of the missions.

ESA's Comet Interceptor mission (Snodgrass \& Jones 2019) is scheduled to launch in 2028 and wait at the Earth-Sun Lagrange point L2 for up to 3-5 years until a suitable long-period comet or ISO is identified for a flyby, potentially making the first ever in situ observations of a comet entering the inner solar system for the first time. The LSST's survey power makes it very likely to be the initial discoverer of the eventual target, and the expected discovery at large heliocentric distance will be critical for mission optimization.

\section{CONCLUSIONS AND PROPOSED ACTIONS}

Rubin Observatory and LSST can be transformative for planetary astronomy. An exciting, but non-comprehensive, list of science impacts has been summarized here; additional impacts are outlined in the LSST Solar System Science Collaboration roadmap (Schwamb et al. 2018a) and the LSST Science Book (LSST Science Collaboration et al. 2009). The LSST will significantly increase the discovery rates of new objects in small-body populations from NEOs to TNOs and active objects, and enable large-scale investigations of the physical and dynamical properties of small bodies. In addition, the LSST can support future spacecraft missions by helping to discover primary, flyby, and extended mission targets and providing context to the results of targeted missions.

Effective use of Rubin Observatory for solar system studies is contingent on preparedness now, as well as strong grant support once data begin to flow. At present, no focused programs exist specifically for analysis of LSST solar system data. Unlike NASA or DOE programs which typically come with a science team, funding, and key projects to be executed, Rubin Observatory is considered a facility, with no established funding for solar system science. This is in marked difference to some international partners who already have preparatory LSST science funding and are gearing up for first 
science. A real risk exists that - having fully funded its $\mathrm{M} \$ 600+$ construction - the U.S. astronomy community may miss out on key LSST discoveries. The Decadal Survey should encourage NSF, NASA, and DOE to develop new Rubin-specific grant programs to ensure this facility fulfils its full potential as a solar system exploration machine. Given that most LSST discoveries will occur during the first few years of the survey, preparedness and early investment will be crucial.

Upon the completion of the 10-year LSST survey, many important additional solar system science investigations will be possible with an extended or "Phase 2" survey. As such, planetary science should be a high-priority consideration in planning the postLSST future of Rubin Observatory.

\section{REFERENCES}

A'Hearn, M. F., et al. 1995, Icarus, 118, 223

Batygin, K., et al. 2019, PhR, 805, 1

Bodewits, D., et al. 2014, Icarus, 229, 190

Brasser, R., \& Wang, J. 2015, A\&A, 573, A102

Brown, M., \& Batygin, K. 2019, AJ, 157, 62

Carvano, J. M., et al. 2010, A\&A, 510, A43

Cochran, A. L., et al. 2015, SSRv, 197, 9

DeMeo, F., \& Carry, B. 2014, Nat., 505, 629

Denneau, L., et al. 2013, PASP, 125, 357

—. 2015, Icarus, 245, 1

Farnocchia, D., et al. 2013, Icarus, 224, 192

Fernández, Y., et al. 2013, Icarus, 226, 1138

Fitzsimmons, A., et al. 2019, ApJL, 885, L9

Giorgini, J. D., et al. 1996, in AAS/DPS

Meeting Abstracts \#28, 25.04

Granvik, M., et al. 2016, Nat., 530, 303

-. 2018, Icarus, 312, 181

Grav, T., et al. 2011, PASP, 123, 423

Heisler, J., \& Tremaine, S. 1986, Icarus, 65, 13

Ishiguro, M., et al. 2016, AJ, 152, 169

Ivezić, V., \& Ivezić, Ž. 2020, arXiv:2007.05600

Ivezić, Ž., et al. 2002, AJ, 124, 2943

Ivezić, Ž., et al. 2019, ApJ, 873, 111

Jewitt, D. 2009, AJ, 137, 4296

Jewitt, D., et al. 2015, Asteroids IV, 221

Jones, R. L., et al. 2018, Icarus, 303, 181

Kaib, N. A., et al. 2011, Icarus, 215, 491

Kavelaars, J., et al. 2009, AJ, 137, 4917

- 2020, The Trans-Neptunian Solar System, 61-77

Kelley, M. S. P., et al. 2019, ApJL, 886, L16

Kokotanekova, R., et al. 2017, MNRAS, 471, 2974

Lamy, P. L., et al. 2004, Comets II, 223
LSST Science Collaboration, et al. 2009, arXiv:0912.0201

Mainzer, A., et al. 2012, ApJ, 745, 7

Mainzer, A. K., et al. 2019, NASA Planetary

Data System

McLoughlin, E., et al. 2015, Icarus, 256, 37

Meech, K. J., et al. 2017, Nat., 552, 378

Moeyens, J., et al. 2020, Icarus, 341, 113575

Morbidelli, A., et al. 2010, AJ, 140, 1391

Nesvorný, D. 2018, ARA\&A, 56, 137

Nesvorný, D., et al. 2017, ApJ, 845, 27

'Oumuamua ISSI Team, et al. 2019, Nat. Ast., 3, 594

Parker, A., et al. 2008, Icarus, 198, 138

Petit, J. M., et al. 2011, AJ, 142, 131

Schwamb, M., et al. 2018a, arXiv:1802.0178

Schwamb, M. E., et al. 2018b, Research Notes of the AAS, 2, 159

Shankman, C., et al. 2017, AJ, 154, 50

Snodgrass, C., \& Jones, G. H. 2019, Nat. Comm., 10, 5418

Snodgrass, C., et al. 2017, A\&A Rv, 25, 5

Solontoi, M., et al. 2010, Icarus, 205, 605

Trilling, D. E., et al. 2018, AJ, 155, 243

Trujillo, C. 2020, The Trans-Neptunian Solar System, 79-105

Trujillo, C., \& Sheppard, S. 2014, Nat., 507, 471

Vereš, P., \& Chesley, S. R. 2017a, AJ, 154, 12

—. 2017b, AJ, 154, 13

Vokrouhlický, D., et al. 2019, AJ, 157, 181

Walsh, K. J., et al. 2011, Nat., 475, 206

Wiegert, P., \& Tremaine, S. 1999, Icarus, 137, 84

Yoshida, F., et al. 2019, Planet. Space Sci., 169, 78 